\title{
Chlorthalidone, not hydrochlorothiazide, is the right diuretic for comparison
}

\author{
Ravi Tejraj Mehta ${ }^{*}$, Anil Pareek and Indranil Purkait
}

\begin{abstract}
We have read the study design "Comparison of effects between calcium channel blocker and diuretics in combination with angiotensin II receptor blocker on 24-h central blood pressure and vascular hemodynamic parameters in hypertensive patients: study design for a multicenter, double-blinded, active controlled, phase 4, randomized trial" by Oh GC, et al. with interest. The authors aim to compare the efficacy of amlodipine or hydrochlorothiazide (HCTZ) with an ARB. However, we wish to highlight that chlorthalidone (CTD) is the evidence-based and recommended anti-hypertensive diuretic, and should replace HCTZ in the trial to effectively compare efficacy against the CCB amlodipine.
\end{abstract}

Keywords: Chlorthalidone, Hydrochlorothiazide, Amlodipine, Hypertension, Thiazide diuretics, Calcium channel blockers

\section{Dear Editor.}

We have read with interest the study design by $\mathrm{Oh}$ GC, et al. [1]. The authors aim to compare the efficacy of combination of calcium channel blocker (CCB) or thiazide diuretic with an angiotensin receptor blocker (ARB). However, we wish to highlight that instead of hydrochlorothiazide (HCTZ), chlorthalidone (CTD) is the evidence-based anti-hypertensive diuretic and should be used to effectively compare efficacy against the CCB amlodipine.

1. CTD is structurally and pharmacokinetically distinct from HCTZ with a much longer half-life (40-60 h vs. 3.2-13.1 h) and a wider volume of distribution. Hence, comparing HCTZ to amlodipine (half-life 38-50 h), with such disparity in half-lives, can lead to unsurprising differences in favour of amlodipine. CTD has unique pleiotropic properties, not shared by HCTZ, like inhibition of platelet aggregation and promotion of angiogenesis [2]. These features impart CTD a unique advantage to effectively lower BP as well as improve cardiovascular $(\mathrm{CV})$ outcomes in hypertension [3].

2. CTD has been employed in several major National Institutes of Health (NIH)-funded randomized controlled trials evaluating hard outcomes: the
Hypertension Detection and Follow-Up Program (HDFP), Multiple Risk Factor Intervention Trial (MRFIT), Systolic Hypertension in the Elderly Program (SHEP) and the Antihypertensive and Lipid-Lowering Treatment to Prevent Heart Attack Trial (ALLHAT); and has been repeatedly shown to reduce $\mathrm{CV}$ morbidity and mortality at clinically used doses. On the other hand, HCTZ at the usual prescribed doses (12.5-25 mg/day) has been called a "paltry" antihypertensive, inferior to all other drug classes, and with no published evidence of reducing CV events [4]. Major global hypertension guidelines including latest ones by ACC/AHA [5], CHEP [6] and NICE [7] recommend preferring chlorthalidone (thiazide-like diuretics) over HCTZ and bendroflumethiazide.

3. The authors have listed measurements of ambulatory pulse wave velocity (PWV), and augmentation index (AIx) as secondary endpoints. In a recent randomized, prospective cross-over study, effects of CTD $12.5 \mathrm{mg}$ vs. HCTZ $25 \mathrm{mg}$, both in combination with ARB (Candesartan $8 \mathrm{mg}$ ), on PWV and AIx were compared. After 8 weeks, CTD arm showed a significant reduction in PWV vs. baseline $(p=0.007)$ and HCTZ $(p=0.033)$ [8].

4. In the MRFIT, in the nine clinics where HCTZ was prescribed predominantly initially, there was a $44 \%$ higher CHD mortality, whereas the trend of

\footnotetext{
* Correspondence: ravi.mehta@ipca.com
} Ipca Laboratories Limited, Mumbai, India 
mortality was favorable in the six clinics that primarily used CTD. It was recommended and accepted by the MRFIT Steering Committee to switch all participants from HCTZ to CTD. Later, with CTD, the mortality trend was reversed and the same group had a $28 \%$ lower risk [9]. Similarly, it has been suggested that the findings of Avoiding Cardiovascular Events Through Combination Therapy in Patients Living With Systolic Hypertension (ACCOMPLISH) trial would have been much more compelling had CTD been the agent selected for the diuretic-based regimen [10].

5. In a randomized, multicenter trial, we found losartan/CTD combination to be as effective as losartan/HCTZ in lowering office BP and was well tolerated [11]. In another recent study, we compared CTD with HCTZ by 24-h ambulatory BP monitoring and found that CTD significantly reduced 24-h ABP as well as daytime and nighttime BP. However, no significant 24-h ABP reduction was seen with HCTZ, which merely converted sustained hypertension into masked hypertension [12].

6. CCBs have been shown to be better at reducing central BP and arterial stiffness than thiazide-type diuretics leading the authors to hypothesize that CCB-based combination will be associated with better CV outcomes. However, in ALLHAT study, substantially higher risk of heart failure with amlodipine was found compared to CTD (RR 1.38; 95\% CI, 1.25-1.52); risk being even higher in patients with diabetes (RR 1.42; 95\% CI, 1.23-1.64) [13]. Thus, the CV outcome of the two combinations must be assessed in a long-term study.

To summarize, we urge the authors to re-think on the choice of HCTZ as the comparator diuretic and replace it with CTD, if feasible.

\footnotetext{
Abbreviations

ACC/AHA: American College of Cardiology/American Heart Association; Alx: Augmentation index; ALLHAT: Antihypertensive and Lipid-Lowering Treatment to Prevent Heart Attack Trial; ARB: Angiotensin receptor blocker; CCB: Calcium channel blocker; CHEP: Canadian Hypertension Education Program; CTD: Chlorthalidone; HCTZ: Hydrochlorothiazide; HDFP: Hypertension Detection and Follow-Up Program; MRFIT: Multiple Risk Factor Intervention Trial; NICE: National Institute for Health and Care Excellence; $\mathrm{NIH}$ : National Institutes of Health; PW: Pulse wave velocity; SHEP: Systolic Hypertension in the Elderly Program
}

\section{Acknowledgements}

Not applicable.

\section{Funding}

There is no funding for this work.

Availability of data and materials

Not applicable.

\section{Authors' contributions}

All authors contributed equally to the manuscript. All authors read and approved the final manuscript.

Ethics approval and consent to participate Not applicable.

Consent for publication

Not applicable.

\section{Competing interests}

Authors are affiliated to Ipca Laboratories Limited and are involved in research studies on chlorthalidone.

\section{Publisher's Note}

Springer Nature remains neutral with regard to jurisdictional claims in published maps and institutional affiliations.

Received: 8 January 2018 Accepted: 19 February 2018

Published online: 01 March 2018

\section{References}

1. Oh GC, Lee HY, Chung WJ, Youn HJ, Cho EJ, Sung KC, et al. Comparison of effects between calcium channel blocker and diuretics in combination with angiotensin Il receptor blocker on 24-h central blood pressure and vascular hemodynamic parameters in hypertensive patients: study design for a multicenter, doubleblinded, active-controlled, phase 4, randomized trial. Clin Hypertens. 2017;23:18.

2. Woodman R, Brown C, Lockette W. Chlorthalidone decreases platelet aggregation and vascular permeability and promotes angiogenesis. Hypertension. 2010;56(3):463-70.

3. Sica DA. Chlorthalidone - a renaissance in use? Expert Opin Pharmacother. 2009;10(13):2037-9.

4. Messerli FH, Makani H, Benjo A, Romero J, Alviar C, Bangalore S. Antihypertensive efficacy of hydrochlorothiazide as evaluated by ambulatory blood pressure monitoring: a meta-analysis of randomized trials. J Am Coll Cardiol. 2011;57(5):590-600.

5. Whelton PK, Carey RM, Aronow WS, et al. 2017 ACC/AHA/AAPA/ABC/ACPM/ AGS/APhA/ASH/ASPC/NMA/PCNA Guideline for the Prevention, Detection, Evaluation, and Management of High Blood Pressure in Adults: A Report of the American College of Cardiology/American Heart Association Task Force on Clinical Practice Guidelines. Hypertension. 2017. Epub ahead of print. https://doi.org/10.1161/HYP.0000000000000065.

6. Leung AA, Daskalopoulou SS, Dasgupta K, et al. Hypertension Canada's 2017 guidelines for diagnosis, risk assessment, prevention, and treatment of hypertension in adults. Can J Cardiol. 2017;33(5):557-76.

7. National Clinical Guideline Centre (UK). Hypertension: the clinical Management of Primary Hypertension in adults: update of clinical guidelines 18 and 34. London: Royal College of Physicians (UK); 2011.

8. Kwon BJ, Jang SW, Choi KY, et al. Comparison of the efficacy between hydrochlorothiazide and chlorthalidone on central aortic pressure when added on to candesartan in treatment-naïve patients of hypertension. Hypertens Res. 2013;36(1):79-84.

9. Multiple Risk Factor Intervention Trial Research Group. Mortality after 10 1/2 years for hypertensive participants in the Multiple Risk Factor Intervention Trial. Circulation. 1990:82(5):1616-28.

10. Ernst ME, Carter BL, Basile JN. All thiazide-like diuretics are not chlorthalidone: putting the ACCOMPLISH study into perspective. J Clin Hypertens (Greenwich). 2009;11(1):5-10.

11. Pareek A, Basavanagowdappa $\mathrm{H}$, Zawar $\mathrm{S}$, et al. A randomized, comparative study evaluating the efficacy and tolerability of losartan-low dose chlorthalidone (6.25 mg) combination with losartan-hydrochlorothiazide $(12.5 \mathrm{mg}$ ) combination in Indian patients with mild-to-moderate essential hypertension. Expert Opin Pharmacother. 2009;10(10):1529-36.

12. Pareek AK, Messerli FH, Chandurkar NB, et al. Efficacy of low-dose Chlorthalidone and hydrochlorothiazide as assessed by 24-h ambulatory blood pressure monitoring. J Am Coll Cardiol. 2016;67(4):379-89.

13. ALLHAT Officers and Coordinators for the ALLHAT Collaborative Research Group. Major outcomes in high-risk hypertensive patients randomized to angiotensin-converting enzyme inhibitor or calcium channel blocker vs diuretic: the antihypertensive and lipid-lowering treatment to prevent heart attack trial (ALLHAT). JAMA. 2002;288(23):2981-97. 